\title{
Oxygen Radical
}

National Cancer Institute

\section{Source}

National Cancer Institute. Oxygen Radical. NCI Thesaurus. Code C1421.

Organic or inorganic chemicals that contain an oxygen atom with an unpaired electron.

This unstable electron configuration causes these chemicals to be highly reactive with other molecules. 